\title{
A small molecule kicks repeat expansion into reverse
}

\author{
Michael D. Flower ${ }^{1}$ and Sarah J. Tabrizi ${ }^{1^{*}}$
}

${ }^{1}$ University College London (UCL) Huntington's Disease Centre, Department of Neurodegenerative Disease, UCL Queen Square Institute of Neurology and Dementia Research Centre at UCL, London, UK. *e-mail: s.tabrizi@ucl.ac.uk

\section{Huntington's disease is a severe progressive neurological disorder caused by a CAG repeat expansion in the $H T T$ gene. A small molecule shows therapeutic potential by inducing contraction of these expanded CAG repeats in cell and mouse models of the disease.}

Repetitive DNA sequences are common in our genomes, and their expansion causes over 40 diseases, including Huntington's disease (HD), myotonic dystrophy (DM1), Friedreich's ataxia and fragile $X$ syndrome ${ }^{1}$. The pathogenic repeats are inherently unstable, tending to expand throughout life, often in the tissues most prominently involved, such as the striatum in $\mathrm{HD}^{2}$ and muscle in $\mathrm{DM}^{3}$, and the expansion rate correlates with disease progression ${ }^{4}$. These observations suggest that somatic instability could be fundamentally involved in the pathogenesis and tissue specificity of these diseases. We have known for quite a while that DNA repair is involved in repeat instability since loss of mismatch repair factors reduces somatic expansion in mouse models of repeat diseases, including $\mathrm{HD}^{5-7}, \mathrm{DM} 1^{8}$ and Friedreich's ataxia ${ }^{9,10}$. Recent genome-wide association studies in HD add further support, showing that variants in DNA repair components, such as the nuclease FAN1, the ligase $L I G 1$ and numerous mismatch repair factors, including $M S H 3$, can change the age of onset and rate of disease progression ${ }^{11-15}$. What if we could stop repeat expansion? Though preventing somatic expansion can reduce aggregation of the toxic protein in HD mice ${ }^{6}$, we do not yet know whether it can slow down or prevent these diseases. In this issue, Nakamori et al. ${ }^{16}$ show in cells and mice that the small molecule naphthyridine-azaquinolone $(N A)^{17}$ specifically binds the expanded CAG repeat tracts that cause polyglutamine diseases like HD, preventing further expansion, and instead encouraging them to contract (Fig. 1).

\section{Halting repeat expansion}

Targeting DNA to edit repeat tracts or prevent their expansion is currently an area of huge therapeutic interest. At the moment, we do not know exactly how repeat expansion occurs, but all current models involve slip outs that form when complementary, repeat-containing DNA strands anneal out of register ${ }^{18}$. Such structures could form when DNA is unwound, for example during DNA replication, transcription or repair. In the present study, Nakamori et al. show that NA binds CAG slip outs better the longer they are, but it does not bind CTG slip outs, which suggests specificity for the expanded CAG repeat.

Normally, when DNA repair proteins, including MSH3, encounter these slip outs, they repair them, and it is slippage during this process that is thought to introduce repeat expansions. However, NA binding prevented repair of synthetic CAG slip outs, and with it the expansion-prone slippage. In fibroblasts from HD patients with up to 180 CAG repeats, NA led to a modest negative skew in the distribution of repeat lengths, as measured by small-pool PCR, suggesting it encourages CAG contraction. In cells expressing an artificial 850 CAG tract, NA induced contractions even when proliferation was chemically prevented, but not when transcription was genetically blocked. This suggests that NA prevents error-prone repair of slip outs that form during transcription, at least in this artificial model.

MSH3 identifies base mismatches and slip outs, initiating mismatch repair, and is required for repeat instability in cell and animal models ${ }^{6,7}$. NA did not affect its binding or dissociation from CAG slip 
outs. siRNA-mediated MSH3 knockdown suppressed expansions, as expected, but also prevented NAinduced contraction, demonstrating that the protective, contraction-inducing effect of $N A$ also involves MSH3. NA competed with Replication Protein A (RPA), which stabilizes single-stranded DNA, preventing polymerases from progressing through repeat regions. This suggests that NA might act by preventing repair proteins, like MSH3 and polymerases, from accessing repeats.

Injection of NA into the left striatum of HD mice prevented the expansion that was seen in the opposite, saline-injected striatum, and even induced contractions relative to stable tissues, like the tail. It also reduced HTT aggregation in nuclei of the mice's striatal medium spiny neurons. However, Nakamori et al. did not evaluate whether NA improves motor phenotypes in these mice, which is vital for determining whether the prevention of somatic instability has real therapeutic potential.

\section{Therapeutic potential?}

NA freely entered cells, was not toxic, and did not disrupt HTT expression. Inhibiting mismatch repair is potentially dangerous, as that is how cancers arise in Lynch syndrome. Importantly, Nakamori et al. show that NA has no effect on the repair of single base mismatches, a core function of mismatch repair machinery. To have therapeutic potential, NA must also bind specifically to the pathogenic expanded repeat, and not have off-target effects elsewhere. To this end, Nakamori et al. demonstrated that it did not alter the CAG length of the normal HTT allele or other repeat regions, and while in cells it was associated with some mutations elsewhere in the genome, these were less than has been observed with cancer chemotherapy, and no large rearrangements or strand breaks were seen.

One would envisage treating polyglutamine disease patients early with NA, in order to stabilize or contract their CAG repeat before onset. However, delivery is likely to be a substantial challenge; NA does not appear to freely cross the blood brain barrier, and in mice, repeated intra-striatal injection was required for maximal efficacy. Though the present study goes some way to demonstrating NA's selectivity for pathogenically expanded CAG repeats, it will be vital to show that it is not mutagenic elsewhere in the genome, either by binding DNA or interfering with DNA repair. Finally, while evidence is mounting for a central role of DNA repair-driven somatic instability in repeat expansion diseases, no one has yet demonstrated that stopping repeat expansion actually improves disease phenotypes in animal models. To conclude, NA is an exciting research compound that has taught us much about the mechanisms underlying repeat instability, but there remain significant challenges in using it to treat repeat expansion diseases.

\section{Competing Interests}

M.D.F. has no competing interests. S.J.T. is on scientific advisory boards for Triplet Therapeutics and LoQus therapeutics and has funding in her lab from Vertex pharmaceuticals.

\section{References}

1. Neil, A.J., Kim, J.C. \& Mirkin, S.M. Bioessays 39, doi: 10.1002/bies.201700077 (2017).

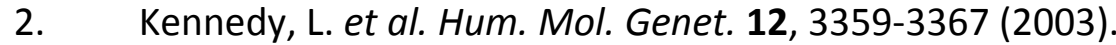

3. Thornton, C.A., Johnson, K. \& Moxley, R.T., 3rd. Ann. Neurol. 35, 104-107 (1994).

4. Ciosi, M. et al. EBioMedicine 48, 568-580 (2019).

5. $\quad$ Pinto, R.M. et al. PLoS Genet. 9, e1003930 (2013).

6. Dragileva, E. et al. Neurobiol. Dis. 33, 37-47 (2009).

7. $\quad$ Tome, S. et al. PLoS Genet. 9, e1003280 (2013).

8. Nakatani, R., Nakamori, M., Fujimura, H., Mochizuki, H. \& Takahashi, M.P. Sci. Rep. 5, 11020 (2015).

9. Bourn, R.L. et al. PLoS One 7, e47085 (2012).

10. Zhao, X.N. et al. Hum. Mol. Genet. 24, 7087-7096 (2015).

11. Genetic Modifiers of Huntington's Disease (GeM-HD) Consortium. Cell 162, 516-526 (2015).

12. Lee, J.M. et al. Hum. Mol. Genet. 26, 3859-3867 (2017).

13. Moss, D.J.H. et al. Lancet Neurol. 16, 701-711 (2017).

14. Genetic Modifiers of Huntington's Disease (GeM-HD) Consortium. et al. bioRxiv 529768 (2019). 
15. Bettencourt, C. et al. Ann. Neurol. 79, 983-990 (2016).

16. Nakamori, M. et al. Nat. Genet. in press (2020).

17. Hagihara, M. \& Nakatani, K. Nucleic Acids Symp. Ser. (Oxf), 147-148 (2006).

18. Axford, M.M. et al. PLoS Genet. 9, e1003866 (2013).

Figure 1 | Naphthyridine-azaquinolone (NA) induces contraction of CAG repeats in a mouse model of Huntington's disease. Stopping or reversing the process of CAG repeat expansion has potential to delay disease onset and reduce disease progression and severity. (Figure concept and design courtesy of Christopher Pearson.) 